\title{
Video compression based on Multiwavelet and Multi Stage Vector Quantization using Adaptive Diamond Refinement Search Algorithm
}

\author{
N. Sardar Basha, A. Rajesh
}

\begin{abstract}
Due to the advances in the digital technology, multimedia processing has become the essential requirement in many applications. These applications find wide use in mobile, personal computer(PC), TV, surveillance and satellite broadcast. Also it is necessary that the video coding algorithms to be updated in order to meet the requirements of latest hardware devices. The processing speed and bandwidth are essential parameters in these applications. A good video compression standard can achieve these parameters adequately. In the proposed system, the video coding standard is implemented using the three important stages. In which the first sage uses multiwavelets to achieve good compression rate. Also it reduces the memory and bandwidth requirement. Second stage is the Multi Stage Vector Quantization(MVSQ) which reduces the complexity of searching process and the size of codebook. Third stage uses Adaptive Diamond Refinement Search(ADRS) algorithm for the motion estimation which has better performance than the Adaptive Diamond Orthogonal Search(ADOS) and Diamond Refinement Search(DRS) algorithms. The combination of multiwavelet, Multi Stage Vector Quantization(MVSQ) and Adaptive Diamond Refinement Search(ADRS) algorithm gives the high compression ratios. Preliminary results indicate that the proposed method has good performance in terms of average number of search points, PSNR values and compression rates.
\end{abstract}

Index Terms-Video coding, video compression, multiwavelets, Multi Stage Vector Quantization, Adaptive Diamond Refinement Search algorithm and MATLAB.

\section{INTRODUCTION}

Now a day's video has become an essential part of entertainment and communication. But the requirement storage space and transmission bandwidth increases with the resolution of video. As an example, the High Definition(HD) videos requires more storage space and bandwidth than the Standard Definition(SD) videos. In this way, video compression helps to reduce the size and thus saves the transmission bandwidth without affecting the display quality of the video on low resolution devices.

Revised Manuscript Received on

N. Sardar Basha, Department of Electronics and Communication Engineering, Sri Chandrasekharendra Saraswathi Viswa Mahavidyalaya University, Kanchipuram, Tamil Nadu, India, sardarbashame@gmail.com
A. Rajesh, Department of Computer Science Engineering, C Abdul Hakeem College of Engineering \& Technology, Vellore, Tamil Nadu, India, amrajesh73@gmail.com

Many applications use the video coding standards (MPEG1, MPEG2, H.263, H.264)[1-3] widely in portable systems like hand-held videophone and multimedia devices. The objective of these video coding standards is to reduce the memory and bandwidth requirements by employing the compression techniques. These compression techniques mainly reduce the redundant data from the video file without disturbing more of its quality. There are different video compression techniques like Discrete Cosine Transform(DCT)[4,5] and Discrete Wavelet Transforms(DWT)[6]. A variant of DCT in integer arithmetic format is used in H.264 which is called as Integer DCT. The problem associated with the DCT is the introduction of annoying blocking artifacts at the boundaries while performing the correlation on the image blocks. This can be overcome by using the wavelets instead of DCT. DWT is a mathematical tool for decomposing an image, hierarchically. A natural alternative for highly scalable video coding involves the use of wavelets. Wavelet transform provides both frequency and spatial description of an image by dividing it into four non-overlapping sub-bands.

\section{LITERATURE REVIEW}

A video coding method using the multiwavelets was proposed by Sudhakar and Jayaraman[7]. This method uses multiwavelets, Kite Cross Diamond Search(KCDS) algorithm for block matching and a combination of Set Partitioning In Hierarchical Trees(SPIHT) algorithm and Set Partitioning in Embedded Block Coding(SPECK). Inherently it uses the wavelets and filters based approach to achieve the better compression performance. Multiwavelets satisfy different properties like orthogonality, symmetry and higher approximation order whereas scalar wavelets couldn't satisfy all these properties simultaneously. This method has also achieved a good compression ratio of $79.07 \%$ with an average Peak Signal to Noise Ratio(PSNR) of $38.84 \mathrm{dBs}$.

Image compression using multiwavelet transform was implemented by V. priya and Ananthi [8]. The multiwavelet systems are Geronimo-Hardin-Massopust(GHM), Chui Lian (CL) and Hardin
Marasovich(HM). SPIHT is

Retrieval Number: B10410682S519/2019@BEIESP DOI: 10.35940/ijrte.B1041.0782S519

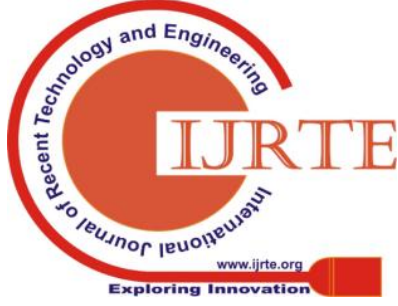

Blue Eyes Intelligence Engineering \&

Sciences Publication 
used for quantization process. A comparison was made among these multiwavelet systems. And the PSNR and MSE of CL are better than GHM and HM multiwavelets respectively.

Lossy image compression using Multiwavelets was proposed by Raj kumar and T. Arivoli [9]. In this implementation, Integer multiwavelets(IMWT) are used for lossy image compression. Using these wavelets, a good compression performance was achieved. These IMWT coefficients are coded using the Magnitude Set(MS) coding and run length encoding methods. In the MS coding, the information will not be considered. Bit plane with zero thresholds are used for coding the sign. The advantage of using the bit planes is to reduce the bpp. In this implementation, it has been achieved a PSNR of up to $40.85 \mathrm{~dB}$.

Vector quantization is widely used in many high-quality and high-rate data compression applications [10]. When the size of codebook increases, it will be difficult to search a code word. This problem has been solved by a new method of quantization called as Multi-Stage Vector Quantization (MSVQ). The computation complexity has been reduced to a great extent using MSVQ. The traditional full search vector quantization(FSVQ)[11] can give a desirable quality for image coding but it has more computational complexity. To overcome this problem, an multistage vector quantization was proposed. Although it can reduce the computational complexity, but it has the problem of coding performance. In order to achieve a good trade-off between these to parameters a new method of quantization was proposed which is called as classified multistage vector quantization (CMSVQ) has been proposed by meng wang, Hui-ping et al.

Motion Estimation is an important step in every video coding method. In these methods, motion vectors are computed by using the block matching algorithms. Diamond Search(DS) is one of the most efficient block matching motion estimation (BMME) algorithms. The problems associated with diamond search are in terms of more number of average search points and its computational complexity. These can be overcome using the Adaptive Diamond Orthogonal Search (ADOS) [12-14]. It uses three different search patterns like Hexagonal Search, Diamond Search and Orthogonal Search.

\section{BLOCK DIAGRAM}

The proposed video encoder is based on the following main modules.

A. Multiwavelet Transform

B. Multi stage vector quantization

C. Adaptive diamond refinement search

The block diagram of video encoder is shown in fig. 3.1.

Retrieval Number: B10410682S519/2019@BEIESP DOI: 10.35940/ijrte.B1041.0782S519

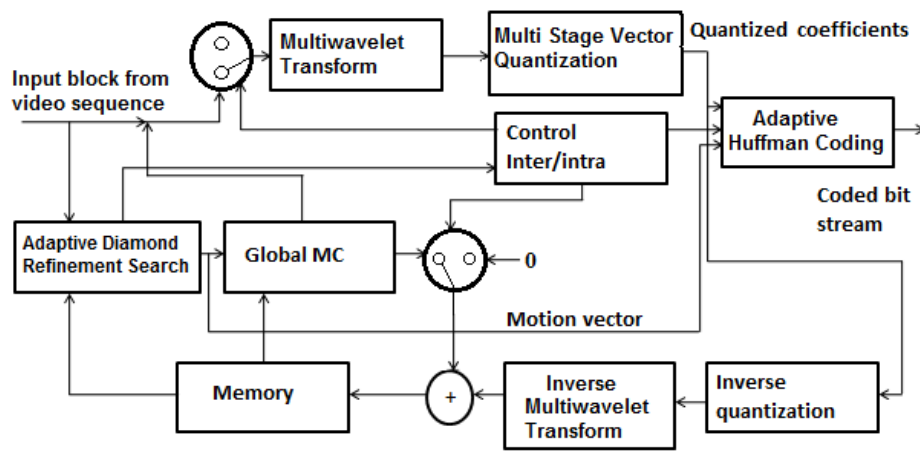

Fig 3.1: Video Encoder

\section{A. Multiwavelet Transform}

Multiwavelets are defined using several wavelets with several scaling functions [07]. These wavelets have the advantages like compact support, orthogonality, symmetry, high order approximation, perfect reconstruction and a good performance at the boundaries. Thus, multiwavelets can perform better than the scalar wavelets. Multiwavelets have two or more scaling functions and wavelet functions.

Here, scaling functions set $\Phi(t)$ is given as below [07]

$\Phi(t)=\left[\Phi_{1}(t), \Phi_{2}(t), \ldots . . \Phi_{r}(t)\right]^{T}$

$\Phi(t) \rightarrow$ multi-scaling function

$r \rightarrow$ mutliplicity of multiwavelet function

Similarly, multiwavelet function is given as below

$\psi(t)=\left[\psi_{l}(t), \psi_{2}(t), \ldots \ldots \psi_{r}(t)\right]^{T}$

Multiwavelets are considered as the vector quantities.

Also these functions can be written using dilation operations as below

$\Phi(t)=\sqrt{2} \sum_{m=-\infty}^{\infty} H_{\mathrm{m}} \Phi(2 t-m)$

$\psi(t)=\sqrt{2} \sum_{m=-\infty}^{\infty} G_{\mathrm{m}} \Phi(2 t-m)$

$\mathrm{H}_{\mathrm{m}}, \mathrm{G}_{\mathrm{m}}$ are called as the filter coefficients. Its size is $\mathrm{NxN}$ matrixes. These multiwavelets are described by the filter banks. Multiwavelets are implemented with the help of multirate filter bank.

As an example a 4-coefficients filter bank uses consists of 4 $\mathrm{NxN}$ matrices ("C") for the low pass filtering. Unlike the scalar wavelet the remaining $4 \mathrm{NxN}$ high pass filter matrices(" D" )

Published By:

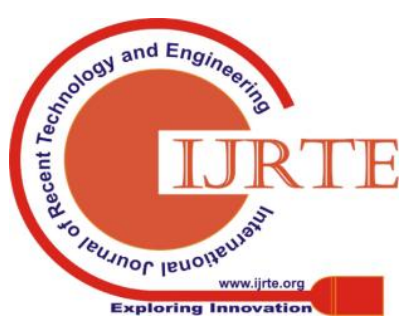

Blue Eyes Intelligence Engineering \&

Sciences Publication 
couldn't be obtained directly by flipping the low pass filter matrices, they must be designed. This filter bank operates on $\mathrm{N}$ input streams (" $\mathrm{x}$ ") and generates $2 \mathrm{~N}$ output streams. These $2 \mathrm{~N}$ outputs can be down sampled by 2 to generate $\mathrm{N}$ outputs.

Figure 3.2 shows the multirate filter bank structure.

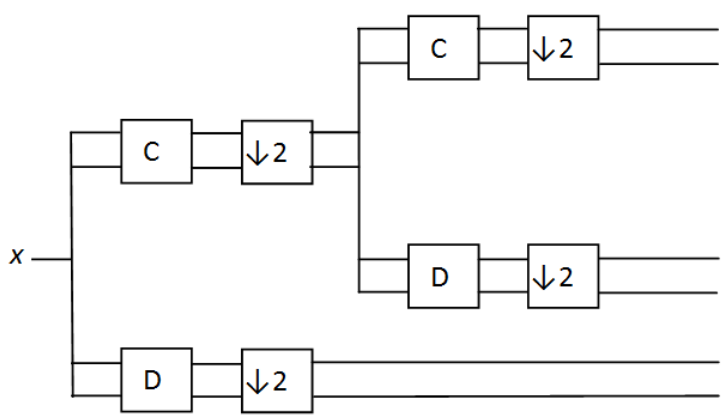

Fig. 3.2: Multirate filter bank structure

\section{B. Multi Stage Vector Quantization}

Multi stage vector quantization consists of $\mathrm{n}$ number of different stages for vector quantization [15]. The very first stage performs a relatively crude quantization of inputs. The remaining stages will operate on the error vectors. These error vectors of each stage are obtained by calculating the difference between the input vector and quantized output vector of its predecessor's stage. The index of the optimal code vector and the label of the output point of the vector quantizer are transmitted to the decoder. The reconstruction is obtained by taking the sum of the output points in each stage. The reconstructed vector is the sum of the output points of each of the stages. For an example in an MVSQ with m-number of stages, the codebook size of the $k^{\text {th }}$ stage quantizer is $\mathrm{N}_{k}$, then the overall codebook size is $\mathrm{N}=\mathrm{N}_{1} \times \mathrm{N}_{2} \ldots . \mathrm{N}_{\mathrm{m}}$.

Also in MVSQ,

The total search complexity $\propto N$

\section{Adaptive Diamond Refinement Search}

Adaptive Diamond Refinement Search is based on combination of Hexagonal Search(HS), Diamond Refinement Search(DRS) and Orthogonal Search(OS) to locate and refine the search process. Hexagonal search finds the best matching point whereas DRS and OS are used to refine the search process. ADRS algorithm consists of the following steps. Corresponding flowchart is shown in fig. 3.3.

\section{Algorithm:}

I: Calculate Sum of Absolute Difference(SAD1) between current block and the reference block. Also if $\mathrm{SAD} 1<512$ (threshold) then $\mathrm{MV}=[00]$ and stop searching.

II. Hexagonal search is performed to calculate the SAD of all individual blocks. Find the minimum of SAD.

III. If the Min(SAD) is less than SAD1 then Diamond Refinement search is performed to find the final MV.

IV. If the Min(SAD) is in between to SAD1 and SAD1+512 then Orthogonal Search is performed to calculate the final motion vector.

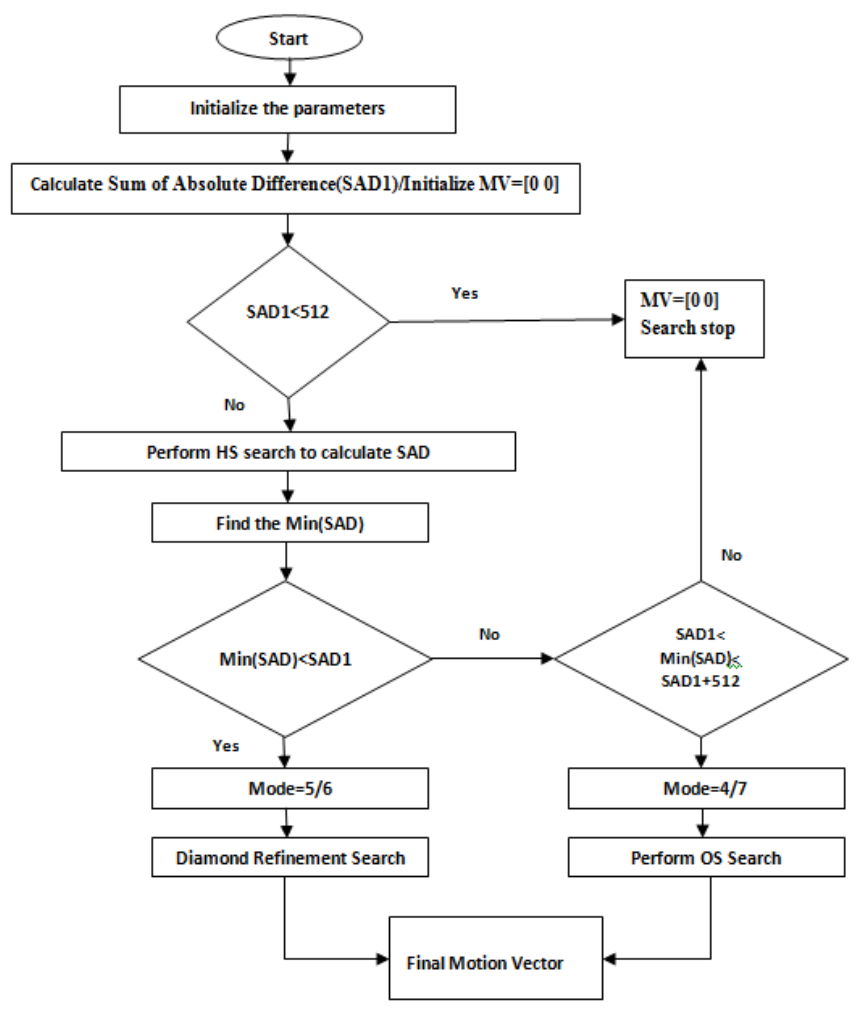

Fig. 3.3: Adaptive Diamond Refinement Search flow chart

\section{EXPERIMENTAL RESULTS}

The proposed system is simulated using the MATLAB programming tool. Simulation is performed on 4 different types of video sequences. Figure 4.1 to 4.3 shows the simulation results of the proposed video compression system.

Published By:

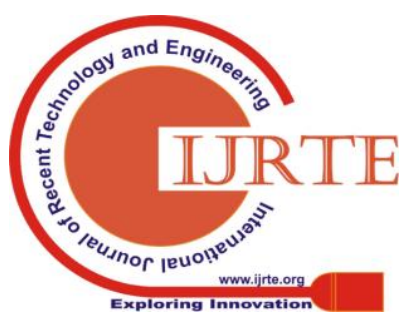

Blue Eyes Intelligence Engineering \&

Sciences Publication 
Video compression based on Multiwavelet and Multi Stage Vector Quantization using Adaptive Diamond Refinement Search Algorithm

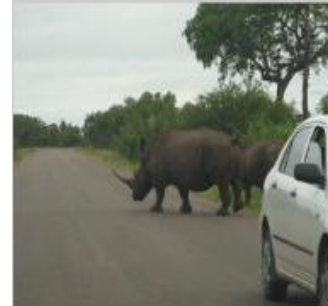

(a)

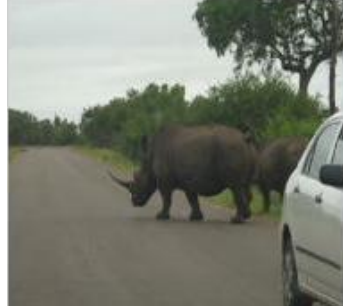

(b)

\begin{tabular}{|c|l|c|c|c|}
\hline & & Search & & system \\
\hline 1 & Akiyo & 13.01 & 5.03 & 5 \\
\hline 2 & $\begin{array}{l}\text { Coast } \\
\text { guard }\end{array}$ & 13 & 5.42 & 5.10 \\
\hline 3 & Foreman & 19.63 & 8.21 & 7.92 \\
\hline 4 & Rhino & 15.01 & 6.02 & 5.88 \\
\hline
\end{tabular}

Fig 4.1: Rhino video sequence with reference and current frames ( $\mathrm{a}$ and $\mathrm{b}$ respectively)

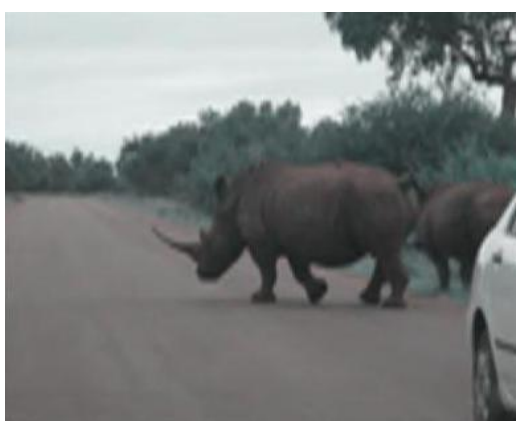

Fig. 4.2: Compressed frame using Multiwavelet Transform and MSVQ

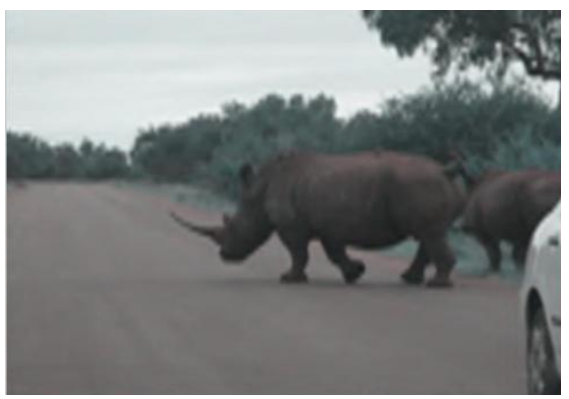

Fig. 4.3: Results of motion compensation using ADRS method

The performance parameters like average number of search points and PSNR of different systems like Diamond Search(DS), Adaptive Diamond Optimization Search were compared with our proposed system(ADRS) are shown in the table 4.1 and 4.2 respectively. And its graphical representation is shown in fig. 4.4 and 4.5 respectively

Table I: Average no. of search points per block frame

\begin{tabular}{|c|c|c|c|c|}
\hline \multirow{2}{*}{$\begin{array}{c}\text { Sr. } \\
\text { No. }\end{array}$} & \multirow{2}{*}{ Videos } & \multicolumn{3}{|c|}{ Algorithm } \\
\cline { 3 - 5 } & & Diamond & ADOS & Proposed \\
\hline
\end{tabular}

Retrieval Number: B10410682S519/2019@BEIESP
Table II: PSNR(dB) comparison of DS, ADOS and proposed systems

\begin{tabular}{|c|l|c|c|c|}
\hline \multirow{2}{*}{$\begin{array}{c}\text { Sr. } \\
\text { No. }\end{array}$} & Videos & $\begin{array}{c}|c| \\
\text { Diamond } \\
\text { Search }\end{array}$ & ADOS & $\begin{array}{c}\text { Proposed } \\
\text { system }\end{array}$ \\
\hline 1 & Akiyo & 43.51 & 43.51 & 51.21 \\
\hline 2 & $\begin{array}{l}\text { Coast } \\
\text { guard }\end{array}$ & 28.34 & 28.34 & 50.01 \\
\hline 3 & Foreman & 23.98 & 23.98 & 35.13 \\
\hline 4 & Rhino & 33.14 & 33.16 & 44.55 \\
\hline
\end{tabular}

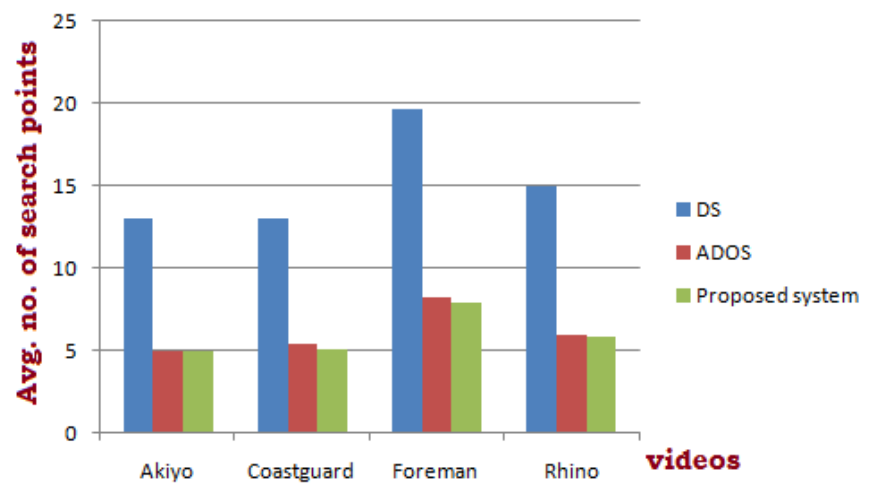

Fig. 4.4: Comparison of average no. of search points per block frame

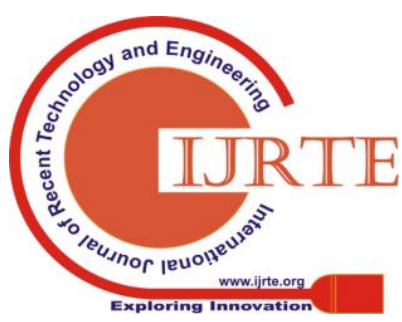

Blue Eyes Intelligence Engineering \&

Sciences Publication 


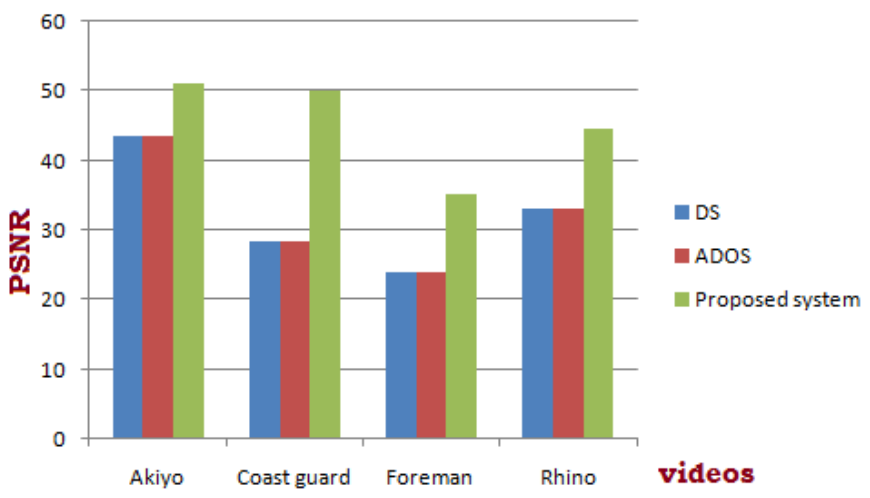

Fig. 4.5: PSNR comparison of different systems with our proposed system

\section{CONClusion}

In this system, multiwavelet transforms are applied on the input frames to reduce the spatial redundancy. These wavelets have the advantages like perfect reconstruction, good performance at the boundaries and high order approximation. In quantization process, Multi Stage Vector Quantization(MSVQ) is adopted to reduce the complexity of searching process and the size of codebook. Adaptive Diamond Refinement Search(ADRS) algorithm is used for the motion estimation which has better performance than the Adaptive Diamond Orthogonal Search and Diamond Refinement Search algorithms. Also this algorithm gives more accurate motion vector prediction than the other algorithms. The different algorithms have been compared for the performance parameters like average no. of search points $(5$ minimum), PSNR values(51.21dB) and compression rate. And, it has been proved that the proposed algorithm has better performance than the other algorithms.

\section{REFERENCES}

[1]. S. Liu, " Performance comparison of MPEG1 and MPEG2 video compression standards," COMPCON 96. Technologies for the Information Superhighway Digest of Papers, p.p. 0199 - 0203.

[2]. Lehtoranta, and Sarinen, " Real-time H.263 encoding of QCIF-images on TMS320C6201 fixed point DSP," IEEE International Symposium on Circuits \& Systems. Emerging Technologies for the 21st Century, 2000, p.p. 0583-0586, vol. 01.

[3]. P. K. Srivastava and Jakkani, " FPGA Implementation of Pipelined $8 \times 8$ 2-D DCT and IDCT Structure for H.264

Protocol," 3rd International Conference for Convergence in Technology, 2018, p.p. 01- 06.
[4]. Ying-Jui Chen, Soontorn Oraintara and Truong Nguyen, "Video compression using integer DCT," Proceedings International Conference on Image Processing, 2000, p.p. 0844-0845, vol. 02.

[5]. Zixiang Xiong, Ramchandran, "A comparative study of DCT- and wavelet-based image coding," IEEE Transactions on Circuits \& Systems for Video Technology, vol. 09, no. 05, p.p. 0692-0695, 1999.

[6]. Bystrov, Gryzov, "Wavelet-Based Video Coding: Modem Implementations and Prospects of Coding Efficiency Increase," IVth International Conference on Engineering \& Telecommunication, 2017, p.p. $038-041$.

[7]. Sudhakar, Jayaraman, "A New Video Coder using Multiwavelets," 2007 International Conference on Signal Processing, Communications and Networking, Chennai, 2007, p.p. 0259- 0264.

[8]. Priya and Ananthi, "Image compression using multiwavelet transform for medical image," International Conference on Innovations in Green Energy and Healthcare Technologies, 2017, p.p. 01- 03

[9]. Rajakumar and Arivoli, "Lossy image compression using multiwavelet transform coding," International Conference on Information Communication and Embedded Systems, 2014, p.p. 01- 06.

[10]. G. Jeong and Lee, "Wavelet-based ECG compression using dynamic multi-stage vector quantization," 4th IEEE Conference on Industrial Electronics and Applications, 2009, p.p. 02100- 02105.

[11]. M. Wang, Zhou, "An Improved Multi-stage Vector Quantization for Image Coding," $\quad 3^{\text {rd }}$ International Conference on Intelligent Information Hiding \& Multimedia Signal Processing, 2007, p.p. 04150420 .

[12]. N. A. Hamid and Sulaiman, "Adaptive Diamond Orthogonal Search Algorithm for Motion Estimation," International Conference on Computer, Communications, and Control Technology, 2015, p.p. 04980501.

[13]. Y. Lai and Lien, "Fast Motion Estimation Based on Diamond Refinement Search for High Efficiency Video Coding," IEEE International Conference on Consumer Electronics, 2019, p.p. 01- 02.

[14]. A. A. Devi, Sumalatha, Priya, Sukruthi and Minisha, " Modified diamond-square search technique for efficient motion estimation," International Conference on Recent Trends in Information Technology, 2011, p.p. 01149- 01153.

[15].Hong Chae and Barnwell, " Fast codeword search for vector quantization using a multi-stage approach," IEEE International Conference on Acoustics, Speech \& Signal Processing. Proceedings, 2000, p.p. 02629- 02632, vol. 05.
Published By:

Blue Eyes Intelligence Engineering \&

Sciences Publication 